\title{
Produtos naturais ativadores de PPAR e marcadores associados ao processo inflamatório na Síndrome Metabólica
}

\author{
BRITO, L.F. ${ }^{*} ;$ TOLEDO, R.C.L. ${ }^{1}$; CARVALHO, I.M.M.'; LEITE, J.P.V.'; RIBEIRO, S.M.R. ${ }^{2 ;}$ PELUZIO, M.C.G. ${ }^{2}$; \\ QUEIROZ, J.H. ${ }^{1}$ \\ ${ }^{1}$ Departamento de Bioquímica e Biologia Molecular; ${ }^{2}$ Departamento de Nutrição e Saúde; Universidade Federal \\ de Viçosa, Av, PH Rolfes, s/n, Cep: 36571-001, Viçosa-Brasil. *larissafroede@yahoo.com.br
}

\begin{abstract}
RESUMO: O processo inflamatório é o elo entre a síndrome metabólica e as doenças cardiovasculares. Para verificar a presença e o grau da inflamação, vários biomarcadores têm sido propostos e investigados. Este trabalho tem como objetivo revisar as recentes pesquisas que associam alguns marcadores expressos no tecido adiposo, enfatizando, dentre eles, a adiponectina, a resistina, a leptina e o transportador de glicose GLUT-4 na síndrome metabólica, a relação da inflamação decorrente desse conjunto de desordens metabólicas sob os receptores proliferadores peroxissomais (PPARs), bem como o efeito de diferentes extratos vegetais e produtos naturais bioativos na ativação desses receptores.
\end{abstract}

Palavras chave: Síndrome Metabólica, processo inflamatório, tecido adiposo, adipocinas.

\begin{abstract}
The inflammatory process in the metabolic syndrome: PPAR activators of natural products and markers associated with metabolic disorders. The inflammatory process is the link between metabolic syndrome and cardiovascular diseases. To verify the presence and degree of inflammation, several biomarkers have been proposed and different receptors have been investigated. This study aims to review recent researches involving some markers expressed in the adipose tissue, emphasizing, among them, adiponectin, resistin, leptin and glucose transporter GLUT-4 in the metabolic syndrome, the relationship of inflammation arising from this set of metabolic disorders on the peroxisome proliferator receptors (PPARs) and the effect of different bioactive compounds in the activation of these receptors.
\end{abstract}

Keywords: Metabolic syndrome, inflammation, adipose tissue, adipokines.

\section{INTRODUÇÃO}

A Síndrome Metabólica (SM) abrange uma grande variedade de alterações fisiopatológicas, todas de repercussões sistêmicas, acometendo os mais distintos órgãos, nos quais são observadas diferentes alterações nos marcadores expressos em diferentes tecidos, como por exemplo, no tecido adiposo (HUANG et al., 2009).

Os receptores proliferadores peroxissomais (PPARs), têm sido estudados nos últimos anos pelo número crescente de evidências demonstrando importante papel na diferenciação dos adipócitos, metabolismos glicídico e lipídico, além do controle vascular. São conhecidos três tipos de PPAR: alfa, gama e beta (teta), sendo que o PPARã é o regulador-chave da diferenciação dos adipócitos, com estímulo para aumento ou diminuição da expressão de vários genes. Os efeitos benéficos de agonistas dos PPARs em diferentes funções já foram demonstrados em estudos clínicos em portadores de doença coronariana, melhorando o perfil metabólico e inflamatório (BAHIA et al., 2006).

O uso clínico de agonistas de PPARs como fibratos e tiazolidinedionas (TZDs) sugere que a ativação de PPAR pode conter o processo inflamatório envolvido no conjunto de desordens metabólicas envolvidas na SM. As ações benéficas e os efeitos indesejáveis provocados pelos fibratos, TZDs e por outros ligantes sintéticos em avaliações clínicas têm fortalecido a relevância clínica dos PPARs, e contribuindo com o aumento em pesquisas com foco em substâncias com propriedades mais seletivas e menos tóxicas (BROWN; PLUTZKY, 2007).

A busca por compostos bioativos, a partir de plantas medicinais, que regulam a atividade transcricional é bastante recente. Nos últimos anos, agonistas e antagonistas de PPARs foram isolados a partir de espécies vegetais. A atividade de diferentes 
extratos também tem sido explorada (HUANG et al., 2005a).

Nesse sentido, essa revisão aborda o processo inflamatório decorrente da síndrome metabólica, marcadores envolvidos nesse conjunto de desordens fisiológicas, bem como compostos bioativos vegetais que atuam como agonista dos receptores PPARs na mediação da inflamação.

\section{Síndrome metabólica e Inflamação}

A Síndrome Metabólica (SM) é vista atualmente como uma epidemia mundial, com números alarmantes, associada à alta morbimortalidade cardiovascular e elevado custo sócioeconômico (FILHO et al., 2006). Reaven (1988), o primeiro a relatar essa associação de fatores, descreve que a síndrome metabólica consiste de obesidade, resistência à insulina, hipertensão, diminuição da tolerância à glicose ou diabetes, hiperinsulinemia, e dislipidemia caracterizada pela elevação plasmática de triacilgliceróis e diminuição da concentração de high-density lipoprotein (HDL-C). Essas alterações ocorrem devido à associação de diferentes fatores, tais como, influências genéticas (alterações nos receptores, enzimas e sinalização) e ambientais (obesidade, tabagismo, consumo excessivo de gordura saturada e ausência de atividade física) acarretando um conjunto de desordens fisiológicas e desenvolvimento da patologia.

O tecido adiposo é o maior reservatório energético do organismo. Ele é composto por adipócitos, por uma matriz de tecido conjuntivo (fibras colágenas e reticulares), tecido nervoso, células do estroma vascular, nódulos linfáticos, células imunes (leucócitos e macrófagos), fibroblastos e pré-adipócitos (células adiposas indiferenciadas). Os adipócitos são células especializadas no armazenamento de lipídios na forma de triacilgliceróis, e dispõem do conjunto de enzimas e proteínas responsáveis pela síntese, armazenamento e mobilização de ácidos graxos, em resposta a alterações na demanda fisiológica. Esses processos são estritamente regulados por fatores genéticos, nutricionais, hormonais e parácrinos (AHIMA; FLIER, 2000).

Em mamíferos estão presentes dois tipos de tecido adiposo: tecido adiposo marrom (TAM) e tecido adiposo branco (TAB). O TAM está presente durante o desenvolvimento fetal, porém diminui a partir do nascimento durante os primeiros anos de vida. É um tecido que atua no controle da temperatura corporal, por ser especializado na produção de calor (PEREIRA, 2008).

O TAB é um órgão heterogêneo que inclui depósitos múltiplos distintos, tais como depósitos subcutâneo, intra-abdominal e intratorácico. Além do papel importante de reserva energética corporal, o TAB tem funções endócrinas recentemente descobertas, as quais respondem pela secreção de várias moléculas bioativas, chamadas adipocinas. Estas substâncias têm produção e atuação tanto nos próprios adipócitos, quanto em outros tecidos do organismo (HERMSDORFF; MONTEIRO, 2004; SÁNCHEZ-MUÑ̃Z et al., 2005).

O acúmulo de tecido adiposo, principalmente na região abdominal, é fundamental para o desencadeamento da síndrome metabólica. Existe uma associação entre o tecido adiposo e as principais células inflamatórias, levando a um aumento da produção de diferentes mediadores inflamatórios e a maior liberação de ácidos graxos livres (AGL) (LOTTENBERG et al., 2007; LOTTENBERG, 2009). $O$ aumento da concentração plasmática de AGL resulta na indução do estresse oxidativo, inflamação e reatividade vascular junto a resistência à insulina (GUO; TABRIZCHI, 2006). Essa resistência resultante da ativação da lipase hormônio sensível em adipócitos leva a um ciclo vicioso de lipólise, consequentemente, aumento de AGL, resistência à insulina e inflamação (FONSECA-ALANIZ et al., 2007). A Figura 1 mostra o desenvolvimento da síndrome metabólica decorrente do aumento da concentração de ácidos graxos livres.

Dados recentes apontaram uma associação entre a obesidade e o estado inflamatório, mostrando que na obesidade ocorre um aumento significativo nas concentrações plasmáticas de TNF $\alpha$, IL-6 e outros mediadores inflamatórios. $O$ tecido adiposo apresenta a maior expressão desses marcadores (DANDONA et al., 2004).

Juntamente com TNF $\alpha$ e IL-6, que são as citocinas expressas em maior quantidade nos adipócitos, três outras importantes proteínas foram relatadas como marcadores associados à síndrome metabólica: leptina, adiponectina e resistina (FONSECA-ALANIZ et al., 2007).

A leptina está associada a funções como saciedade, inibição da ingestão alimentar, regulador da função sexual e modulador imune, além de induzir a agregação plaquetária. Contudo, em longo prazo, pode contribuir para o estado próinflamatório da obesidade e aterogênese (NAKATA et al., 1999; LA CAVA et al., 2004). Essa proteína é produzida no TAB e demonstra similaridade estrutural com a família de citocinas como a resistina. Ela atua nos receptores expressos no hipotálamo promovendo uma sensação de saciedade e equilibrando o balanço energético. Em situações fisiológicas normais a leptina promove uma melhora na sensibilidade à insulina (hepática e no músculo esquelético) além de modular a função das células $\beta$ pancreáticas (FORNARI, 2009). Tanto a deficiência como a resistência a leptina podem causar o aumento e o 


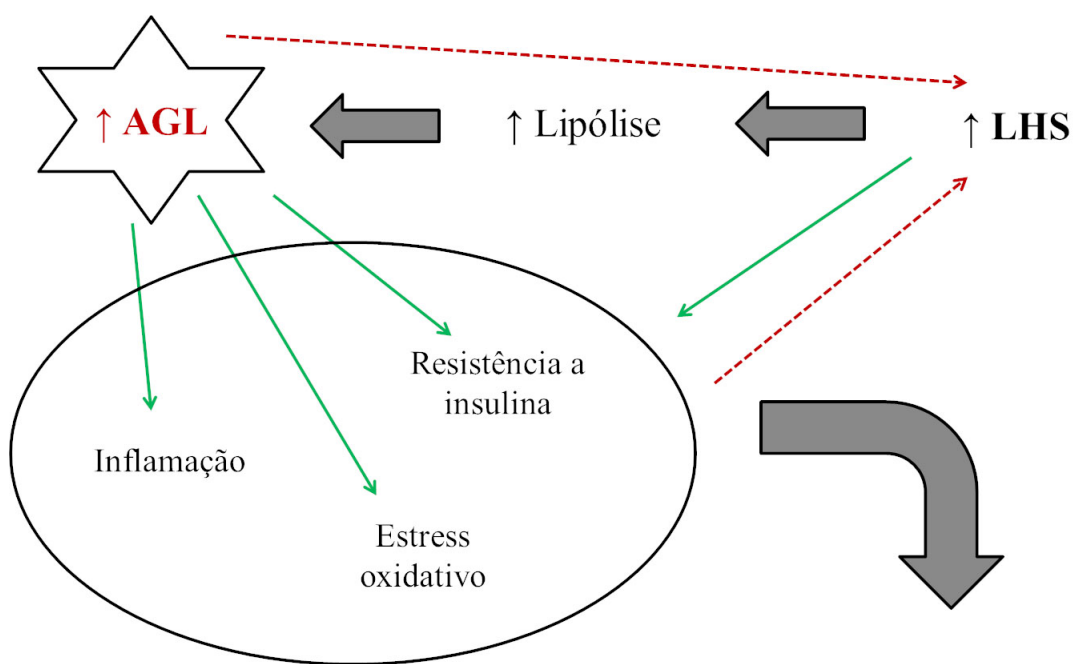

\section{SÍNDROME \\ METABÓLICA}

LHS: Lipase hormônio sensível; AGL: ácidos graxos livres;

Representação do ciclo vicioso de lipólise.

FIGURA 1. Alterações metabólicas decorrentes do aumento de ácidos graxos livres levando à síndrome metabólica.

desenvolvimento da obesidade (RABE et al., 2008).

A adiponectina é secretada pelos adipócitos em indivíduos normais, antinflamatória, antiaterogênica, e, em altas concentrações pode levar ao ganho de peso e à obesidade (KUBOTA et al., 2002; UKKOLA; SANTANIEMIM, 2002). Isso sugere que em elevadas concentrações essas duas proteínas podem ser marcadores da aterosclerose e doenças cardiovasculares (PISCHON et al., 2004). Essa proteína é sintetizada exclusivamente no tecido adiposo, e ao contrário de outras proteínas que são secretadas pelos adipócitos, a expressão diminui à medida que o tecido adiposo aumenta. Sua concentração se encontra diminuída em humanos e camundongos obesos ou portadores de resistência insulínica. Alguns tratamentos nutricionais e terapêuticos que melhoram a sensibilidade à insulina, como por exemplo, a restrição energética, perda de peso e tratamento com glitazones e tiazolidinodionas (TZDs) aumentam a expressão gênica de adiponectina e sua quantidade circulante (MAYNADIER et al. 2009).

A resistina induz à resistência à insulina (PACKER; SIES, 2008). Drogas anti-diabéticas como tiazolidinedionas (TZDs) ou glitazones diminuem a concentração de resistina em humanos, e induzem um aumento da expressão de mRNA da adiponectina principalmente nos adipócitos, aumentando a concentração e a expressão da adiponectina no plasma, mostrando o efeito antinflamatório dessas drogas (HAMMARSTED et al., 2005; GHANIM et al., 2006; MORIUCHI et al., 2007). Ela também é sintetizada pelo TAB, visto que quando administrada intraperitonealmente, eleva os níveis de glicose plasmática e induz a resistência insulínica. Em nível celular, a resistina exerce potente efeito próinflamatório levando ao aumento da produção de citocinas próinflamatórias e moléculas de adesão, contudo, a literatura ainda é controversa (RABE et al., 2008).

Outro importante marcador da síndrome metabólica é o GLUT-4 (HUANG et al., 2009). O GLUT-4 é o chamado transportador de glicose insulino sensível, cujo principal papel é proporcionar a captação de glicose insulino-mediada em tecidos adiposo e muscular. Correlaciona-se de maneira direta com o aumento ou redução da sensibilidade à insulina (ISHIKI; KLIP, 2005; MACHADO et al., 2006).

O GLUT-4 tem sido investigado há mais de uma década em vários modelos experimentais, assim como em humanos com obesidade, resistência à insulina $(\mathrm{RI})$, diabetes e hipertensão, todos componentes da síndrome metabólica (KLOTING et al., 2006). Papa e cols. (2002) revelaram que, durante o processo de desenvolvimento da obesidade, inicialmente, quando o ganho de peso é acelerado, há aumento na sensibilidade à insulina e no GLUT-4 no tecido adiposo; posteriormente, quando a obesidade se estabiliza, sobrevém redução do GLUT-4 no tecido adiposo e a RI se manifesta.

Na síndrome metabólica, em consequência do desequilíbrio no fluxo de ácidos graxos livres, ocorrem modificações na expressão gênica de alguns marcadores, podendo representar uma conexão regulatória entre a adiposidade, síndrome metabólica e doenças cardiovasculares. 


\section{Interesse clínico por ligantes PPARs}

Os receptores nucleares atuam diretamente no processo transcricional, controlando a expressão de genes-alvo que regulam diversos processos biológicos como a reprodução, o desenvolvimento e o metabolismo geral (PEREIRA, 2008).

Uma área importante no campo de pesquisas metabólicas se refere às funções dos receptores nucleares PPARs como alvos terapêuticos para desordens no metabolismo de lipídios e glicose em virtude desses fatores estarem associados a doenças como diabetes, dislipidemia, aterosclerose, obesidade, síndrome metabólica e doenças cardiovasculares (SHEARER; BILLIN, 2007). PPARs apresentam também importante papel na sensibilização periférica à insulina por meio da redução na expressão de resistina e TNFá, aumento da expressão da adiponectina e da atividade da lípase lipoprotéica (LLP) (HERMSDORFF; MONTEIRO, 2004).

O uso clínico de agonistas de PPARs como fibratos e tiazolidinedionas (TZDs), em conjunto com evidências que sugerem que a ativação de PPAR pode conter a evolução de processos inflamatórios e aterosclerose tem instigado o interesse e a busca por novos ligantes. Soma-se a esses fatores os efeitos indesejados provocados pelos fibratos, TZDs e por outros ligantes sintéticos em avaliações clínicas, além dos resultados obtidos em estudos cardiovasculares. Em conjunto, as ações benéficas e os efeitos indesejados provocados pelos fibratos, TZDs e por outros ligantes sintéticos em avaliações clínicas têm fortalecido a relevância clínica dos PPARs, e contribuindo com o aumento em pesquisas com foco em substâncias com propriedades mais seletivas e menos tóxicas (BROWN; PLUTZKY, 2007).

As tiazolidinedionas (TZDs), agonistas de PPARã, são utilizadas clinicamente em tratamento de diabete tipo 2. Agonistas de PPARã também apresentaram efeitos antiaterogênicos em modelos animais. Entretanto, tais substâncias podem promover efeitos colaterais clinicamente significativos como ganho de peso, em função de retenção de líquido e do aumento na adiposidade, ações essas que parecem ser potencializadas quando as TZDs são utilizadas em associação à insulina. Alguns estudos apontam para o aumento dos efeitos da rosiglitazona no risco de infarto de miocárdio e na morte por doenças cardiovasculares, embora essas informações ainda não sejam conclusivas. Efeitos hepatotóxicos têm sido associados ao uso de troglitazona, além de prejuízo no metabolismo ósseo (MICHALIK et al., 2006; BROWN; PLUTZKY, 2007; BERBEROGLU et al., 2007; NISSEN; WOLSKI, 2007).

Além dos benefícios clínicos bem estabelecidos em diabetes e dislipidemia, vários estudos relatam a utilização de ligantes de PPARs na síndrome metabólica. Os ligantes de PPAR, por atuarem na regulação de vias metabólicas envolvidas nas desordens que acompanham a síndrome metabólica, emergem com grande potencial de atuação no tratamento de pacientes com essa complexa rede de alterações fisiológicas (FIÉVET et al., 2006; KASUGA et al., 2006; GURNELL, 2007; WILKINSON et al., 2008).

\section{Ligantes para PPARs de origem natural}

Se por um lado o desenvolvimento de moduladores sintéticos da expressão gênica em níveis transcricionais é realizado há muito tempo, a busca por substâncias isoladas a partir de plantas medicinais que detenham atividade transcricional é mais recente. Nos últimos anos, agonistas e antagonistas dos receptores PPARs foram isolados a partir de extratos vegetais (HUANG et al., 2005b). A atividade dos extratos, de frações de extratos e de substâncias isoladas sobre a ativação transcricional dos receptores PPARs é avaliada basicamente com a realização de ensaios de gene repórter em cultura de células, ensaios de competição pela ligação e teste de diferenciação de adipócitos, sendo que alguns trabalhos recorrem aos testes em animais para correlacionar os efeitos observados in vitro com os efeitos in vivo (PEREIRA, 2008). A tabela 1 mostra o efeito de diferentes extratos e compostos bioativos na ativação dos receptores PPARs.

O conjunto de desordens fisiológicas envolvidas na síndrome metabólica faz com que várias pessoas recorram ao uso de plantas medicinais como alternativa de tratamento. No Brasil, com a diversidade da flora, há um amplo campo de atuação na área de validação das propriedades farmacológicas atribuídas empiricamente às plantas medicinais, principalmente na seleção de ligantes para receptores nucleares, em que as pesquisas ainda são insipientes. Plantas medicinais que comprovadamente reduzam as alterações ocorridas nesse estado patológico têm grande potencial de bioatividade no tratamento da síndrome metabólica. Por essa razão, plantas usadas popularmente pela ação antidiabética, hipolipemiante, antinflamatória, antiobesidade, dentre outras propriedades farmacológicas, vêm sendo utilizadas por grupos de pesquisa no rastreamento de espécies que atuem no metabolismo energético total com o objetivo de identificação de novos agonistas para receptores PPARs.

\section{CONSIDERAÇÕES FINAIS}

O aumento da incidência da síndrome metabólica nos países desenvolvidos e em 


\section{TABELA 1}

\begin{tabular}{|c|c|c|c|}
\hline Família / espécie & Extrato / Princípio ativo & Atividade & Referência \\
\hline \multicolumn{4}{|l|}{ Anacardiaceae } \\
\hline Mangifera indica & Glicosilxantona: mangiferina & $\begin{array}{l}\text { Ativador de PPAR } \alpha . \\
\text { e PPAR } \gamma \text {. }\end{array}$ & $\begin{array}{l}\text { WILKINSON et al., 2008; } \\
\text { KAUSHIK et al., } 2010 .\end{array}$ \\
\hline \multicolumn{4}{|l|}{ Araliaceae } \\
\hline Panax radix palva & Extrato aquoso de raiz de ginseng branco. & Ativador de PPAR $\gamma$. & CHUNG et al., 2001. \\
\hline \multicolumn{4}{|l|}{ Cannabaceae } \\
\hline Cannabis sativa & $\begin{array}{l}\text { Ácido julêmico (análogo sintético do THC) } \\
\text { Cannabidiol }\end{array}$ & $\begin{array}{l}\text { Ativador de PPAR } \gamma \text {. } \\
\text { Ativador de PPAR } \gamma \text {. }\end{array}$ & $\begin{array}{l}\text { LIU et al., 2003;BURSTEIN, } \\
\text { 2005. IZZO et al., 2009; } \\
\text { O'SULLIVAN et al., 2009; } \\
\text { O'SULLIVAN \& KENDALL, } 2009\end{array}$ \\
\hline \multicolumn{4}{|l|}{ Cucurbitaceae } \\
\hline \multirow{2}{*}{$\begin{array}{r}\text { Gynostemma pentaphyllum } \\
\text { Mormodica charantia }\end{array}$} & Gipenosídeo XLIX. & Ativador de PPAR $\alpha$. & HUANG et al., 2006. \\
\hline & $\begin{array}{l}\text { Extrato em acetato de etila do fruto e } \\
\text { compostos isolados. }\end{array}$ & Ativador de PPAR $\alpha$. & $\begin{array}{l}\text { CHAO HUANG, 2003; } \\
\text { CHUANG et al., } 2006 .\end{array}$ \\
\hline \multicolumn{4}{|l|}{ Leguminosae } \\
\hline Astragalus membranaceus & Biocanina A, formononetina. & \multicolumn{2}{|l|}{ Ativador de PPAR $\alpha$ e PPAR $\gamma$. } \\
\hline Glycine max & Fitoestrógeno: genisteína. & Ativador de PPAR $\gamma$. & DANG et al., 2003. \\
\hline Glycyrrhiza uralensis & $\begin{array}{l}\text { Prenilflavonóides: glicicumarina, } \\
\text { glicirina, dehidrogliasperina C, } \\
\text { dehidrogliasperina D. }\end{array}$ & Ativador de PPAR $\gamma$. & KURODA et al., 2003. \\
\hline \multicolumn{4}{|l|}{ Meliaceae } \\
\hline Swietenia mahagony & Extrato etanólico da semente. & Ativador de PPAR $\gamma$. & LI et al., 2005. \\
\hline \multicolumn{4}{|l|}{ Pinaceae } \\
\hline Pseudolarix kaempferi & Ácido pseudolárico B. & Ativador de PPAR $\alpha$. & JARADAT et al., 2003. \\
\hline \multicolumn{4}{|l|}{ Polyporaceae } \\
\hline Poria cocos & Ácido dehidrotrametanólico. & Ativador de PPAR $\gamma$. & SATO et al., 2002. \\
\hline \multicolumn{4}{|l|}{ Ranunculaceae } \\
\hline \multirow[t]{2}{*}{ Clematis pickeringii } & Extrato etanólico do caule e frações. & \multicolumn{2}{|l|}{ Ativador de PPAR $\alpha$} \\
\hline & & e PPAR $\gamma$. & LI et al., 2006. \\
\hline \multicolumn{4}{|l|}{ Rutaceae } \\
\hline Citrus grandis & Flavanona: narenginina. & Ativador de PPAR $\gamma$. & HARMON; HARP, 2001. \\
\hline \multicolumn{4}{|l|}{ Theaceae } \\
\hline Camelia sinensis & $\begin{array}{l}\text { Extrato aquoso de chá verde, chá } \\
\text { preto e galato epigalocatequina. }\end{array}$ & Ativador de PPAR $\alpha$. & LEE, 2004. \\
\hline \multicolumn{4}{|l|}{ Vitaceae } \\
\hline Vitis vinifera & $\begin{array}{l}\text { Terpenóides: farnesol, gerannilgeraniol. } \\
\text { Proantocianidinas derivadas de semente. }\end{array}$ & $\begin{array}{l}\text { Ativador de PPAR } \alpha \text { e PPAR } \gamma \\
\text { Ativador de PPAR } \gamma \text {. }\end{array}$ & $\begin{array}{l}\text { TAKAHASHI et al., } 2002 . \\
\text { MA et al., } 2007 .\end{array}$ \\
\hline
\end{tabular}

THC: tetrahidrocanabinol. Fonte: LEE, 2004; HUANG et al., 2005; CHUANG et al., 2006; HUANG et al., 2006; LI et al., 2006; SHEN et al., 2006; MA et al., 2007; PEREIRA, 2008; HUANG et al., 2009; KAUSHIK et al., 2010. 
desenvolvimento é um dos fatores contribuintes para a expansão de estudos utilizando compostos naturais no tratamento e prevenção de desordens metabólicas desencadeadas no processo inflamatório. Nesse contexto, são necessárias pesquisas que abordem o processo inflamatório decorrente das alterações fisiológicas envolvidas nas diferentes patologias que compõem a síndrome metabólica, e associem os principais marcadores envolvidos nesse conjunto de desordens metabólicas, bem como compostos bioativos vegetais agonistas dos receptores PPARs como coadjuvantes na mediação da inflamação.

\section{AGRADECIMENTOS}

À Fapemig, CNPq e Capes.

\section{REFERÊNCIA}

AHIMA, R.S.; FLIER, J.S. Adipose tisue as na endocrine organ. Trends in Endocrinology \& Metabolism, v. 11, n. 8, p. 327-332, 2000.

BAHIA, L.; AGUIAR, L.G.K.; VILLELA, N.R.; BOTTINO, D.; BOUSKELA, E. O endotélio na Síndrome Metabólica. Arquivos Brasileiros de Endocrinologia \& Metabologia, v. 50, n.2, p. 291-303, 2006.

BERBEROGLU, Z.; GURSOY, A.; BAYRAKTAR, N.; YAZICI, A.C.; TUTUNCU, N.B.; DEMIRAG, N.G. Rosiglitazone decreases serum bone-specific alkaline phosphatase activity in postmenopausal diabetic women. The journal of Clinical Endocrinology \& Metabolism, v. 92, n. 9, p. 3523-3530, 2007.

BROWN, J.F.; PLUTZKY, J. Peroxisome proliferatoractivated receptors as transcriptional nodal points and therapeutic targets. Circulation, v. 115, n. 4, p. 518533, 2007.

BURSTEIN, S. PPARã: A nuclear receptor with affinity for cannabinoids. Life Sciences, v. 77, n. 14, p. 16741684, 2005.

CHAO, C.Y.; HUANG, C.J. Bitter Gourd (Momordica charantia) Extract Activates Peroxisome ProliferatorActivated Receptors and Upregulates the Expression of the Acyi CoA Oxidase Gene in H411EC3 Hepatoma Cells. Jounal of Biomedical Science, v.10, n. 2, p. 782-791, 2003.

CHUANG, C.Y.; HSU, C.; CHAO, C.Y.; WEIN, Y.S.; KUO, Y.H.; HUANG, C.J. Fractionation and identification of 9c, 11t, 13t-conjugated linolenic acid as an activator of PPARá in bitter gourd (Momordica charantia L.). Jounal of Biomedical Science, v. 13, n. 6, p. 763-772, 2006.

CHUNG, S. H.; CHOI, C. G.; PARK, S. Comparisons between White Ginseng Radix and Rootlet for Antidiabetic Activity and Mechanism in KKAy Mice. Archives of Pharmacol Research, v. 24, n.3, p.214218, 2001.

DANDONA, P.; ALJADA, A.; BANDYOPADHYAY, A. Inflammation: the link between insulin resistance, obesity and diabetes. Trends in Immunology, v. 25, n. 1 p. 4-7, 2004.

DANG, Z. C.; AUDINOT, V.; PAPAPOULOS, S. E.; BOUTIN, J. A.; LOWIK, C. W. Peroxisome proliferator-activated receptor gamma (PPARgamma) as a molecular target for the soy phytoestrogen genistein. The Journal of Biological Chemistry, v. 278, n. 1, p. 962-967, 2003. FIEVET, C.; FRUCHART, J.C.; STAELS, B. PPARá and PPARã dual agonists for the treatment of type 2 diabetes and the metabolic syndrome. Current Opinion in Pharmacology, v. 6, n. 6, p. 606-614, 2006.

FILHO, F.F.R.; MARIOSA, L.S.; FERREIRA, S.R.G.; ZANELLA, M.T. Gordura Visceral e Síndrome Metabólica: Mais que uma simples associação. Arquivos Brasileiros de Endocrinologia \& Metabologia, v. 50, n.2, p. 230-238, 2006.

FONSECA-ALANIZ, M.H.; TAKADA, J.; ALONSO-VALE, M.I.C.; LIMA, F.B. Adipose tissue as an endocrine organ: from theory to practice. Jornal de Pediatria, v. 83, n. 5, p. 192-203, 2007.

FORNARI, J.V. Efeito da inibição de TNF-á sobre as alterações hepáticas associadas à obesidade experimental. 2009. 49p. Dissertação de Mestrado. Universidade São Francisco, Bragança Paulista, 2009.

GHANIM, H.; DHINDSA, S.; ALJADA, A.; CHAUDHURI, A.; VISWANATHAN, P.; DANDONA, P. Low dose rosiglitazone exerts an anti-inflammatory effect with an increase in adiponectin independently of free fatty acid (FFA) fall and insulin sensitization in obese type 2 diabetics. The journal of Clinical Endocrinology \& Metabolism, v. 91, n. 9, p. 3553-3558, 2006.

GIRÓN, M.D.; SEVILLANO, N.; SALTO, R.; HAIDOUR, A.; MANZANO, M.; JIMÉNEZ, M.L.; RUEDA,R.; PEDROSA,J.M.L. Salacia oblonga extract increases glucose transporter 4-mediated glucose uptake in L6 rats myotubes: Role of mangiferin. Clinical Nutrition, v. 28, n. 5, p. 565-574, 2009.

GUO, L.; TABRIZCHI, R. Peroxisome proliferator-activated receptor gamma as drug target in the pathogenesis of insulin resistance. Pharmacology Therapeutics, v. 111, n. 1, p. 145-173, 2006.

GURNELL, M. 'Striking the right balance' in targeting PPARã in the metabolic syndrome: novel Insights from human genetic studies. PPAR Research, v. 2007, n.83593, p. 1-14, 2007.

HAMMARSTEDT, A.; SOPASAKIS, V.R.; GOGG, S.; JANSSON, P.A.; SMITH, U. Improved insulin sensitivity and adipose tissue dysregulation after short-term treatment with pioglitazone in non-diabetic, insulin resistant subjects. Diabetologia, v. 48, n. 1, p. 96-104, 2005.

HARMON, A. W.; HARP, J. B. Differential effects of flavonoids on 3T3-L1 adipogenesis and lipolysis. American Journal of Physiology - Cell Physiology, v. 280, n, 4, p. C807-C813, 2001.

HERMSDORFF, H.H.M.; MONTEIRO, J.B.R. Gordura visceral, subcutânea ou intramuscular: onde está o problema? Arquivos Brasileiros de Endocrinologia \& Metabologia, v. 48, n. 6, p. 803-811, 2004.

HUANG, T. H. W.; TEOH, A.W.; LIN, B. L.; LIN, D.S.H.; ROUFOGALIS, B. The role of herbal PPAR modulators in the treatment of cardiometabolic syndrome. Pharmacological Research, v. 60, n. 3, p. 195-206, 2009.

HUANG, T. H-W.; PENGA, G.; KOTA, B.P; LI, G.Q.; YAMAHARA, J.; ROUFOGALIS, B.D.; LI, Y. Anti-diabetic action of Punica granatum flower extract: Activation

Rev. Bras. PI. Med., Campinas, v.15, n.3, p.449-466, 2013. 
of PPARã and identification of an active component. Toxicology and Applied pharmacology, v. 207, n. 2, p. 160-169, 2005b.

HUANG, T.H.W.; LI, Y.; RAZMOVSKI-NAUMOVSKI, V.; TRAN, V.H.; LI, G.Q.; DUKE, C.C.; ROUFOGALIS, B.D. Gypenoside XLIX isolated from Gynostemma pentaphyllum inhibits nuclear factor-kappaB activation via PPAR-alpha-dependent pathway. Journal of Biomedical Science, v. 13, n. 4, p. 535-548, 2006.

HUANG, T.H-W.; KOTA, B. P.; RAZMOVSKI, V.; ROUFOGALIS, B.D. Herbal or Natural Medicines as Modulators of Peroxisome Proliferator-Activated Receptors and Related Nuclear Receptors for Therapy of Metabolic Syndrome. Basic \& Clinical Pharmacology Toxicology, v. 96, n. 1, p. 3-14, 2005a.

ISHIKI, M.; KLIP, A. Minireview: recent developments in the regulation of glucose transporter-4 traffic: new signals, locations, and partners. Endocrinology, v. 146, n. 12, p. 5071-5078, 2005.

IZZO, A.A.; BORRELLI, F.; CAPASSO, R.; DI MARZO, V.; MECHOULAM, R. Non-psychotropic plant cannabinoids: new therapeutic opportunities from an ancient herb. Trends in Pharmacological Sciences, v. 30, n. 10, p. 515-527, 2009.

JARADAT, M. S.; NOONAN, D. J.; WU, B.; AVERY, M. A.; FELLER, D. R. Pseudolaric acid analogs as a new class of peroxisome proliferator-activated receptor agonists. Planta Medica, v. 68, n. 8, p. 667-671, 2003.

KASUGA, J.; YAMASAKI, D.; ARAYA, Y.; NAKAGAWA, A.; MAKISHIMA, M.; DOI, T.; HASHIMOTO, Y.; MIYACHI, $H$. design, synthesis, and evaluation of a novel series of asubstituted phenylpropanoic acid derivaties as human peroxisome proliferator-activated receptor (PPAR) á/ä dual agonists for the treatment of metabolic syndrome. Bioorganic Medicinal Chemistry, v. 14, n. 24, p. 84058414, 2006.

KAUSHIK, G.; SATYA, S.; KHANDELWAL, R.K.; NAIK, S.N. Commonly consumed Indian plant food materials in the management of diabetes mellitus. Diabetes and Metabolic Syndrome: Clinical Research an Reviews, v. 4, n. 1, p. 21-40, 2010.

KLOTING, N.; BLUHER, M.; KLOTING, I. The polygenetically inherited metabolic syndrome of WOKW rats is associated with insulin resistance and altered gene expression in adipose tissue. Diabetes Metabolism Research Review, v. 22, n. 2, p. 146154, 2006.

KUBOTA, N.; TERAUCHI, Y.; YAMAUCHI, T.; KUBOTA, T.; MOROI, M.; MATSUI, J.; ETO, K.; YAMASHITA, T.; KAMON, J.; SATOH, H.; YANO, W.; FROQUEL, P.; NAGAI, R.; KIMURA, S.; KADOWAKI, T.; NODA, T. Disruption of adiponectin causes insulin resistance and neointimal formation. Journal Biology Chemistry, v. 227, n. 29, p. 25863-25866, 2002.

KURODA, M.; MIMAKI, Y.; SASHIDA, Y.; MAE, T.; KISHIDA, H.; NISHIYAMA, T.; TSUKAGAWA, M.; KONISHI, E.; TAKAHASHI, K.; KAWADA, T.; NAKAGAWA, K. K.; KITAHARA, M. Phenolics with PPAR-gamma ligandbinding activity obtained from licorice (Glycyrrhiza uralensis roots) and ameliorative effects of glycyrin on genetically diabetic KK-A(y) mice. Bioorganic \& Med. Chemistry Letters, v. 13, n. 24, p. 4267-4272, 2003.

LA CAVA, A.; ALVIGGI, C.; MATARESE, G. Unraveling the multiple roles of leptin in inflammation and autoimmunity. Journal of Molecular Medicine, v. 82, n. 1, p. 4-11, 2004

LEE, K. Transactivation of peroxisome proliferatoractivated receptor á by Green tea extracts. Journal of Veterinary Science, v.5, n. 4, p. 325-330, 2004.

LI, D. D; CHEN, J.; CHEN, Q.; LI, G.; CHEN, J.; YUE, J.; CHEN, M.; WANG, X.; SHEN, J.; SHEN, X.; JIANG, H. Swietenia mahagony extract shows agonistic activity to PPARã and gives ameliorative effects on diabetic $d b /$ $d b$ mice. You have full text access to this content. Acta Pharmacologica Sinica. v.26, n.2, p. 220-222, 2005.

LI, R.W.; LIN, G.D.; LEACH, D.N.; WATERMAN, P.G.; MYERS, S.P. Inhibition of COXs and 5-LOX and activation of PPARs by Australian Clematis species (Ranunculaceae). Journal of Ethnopharmacology, v. 104, n. 1-2, p. 138-143, 2006.

LIU, J.; LI, H.; BURSTEIN, S.H.; ZURIER, R. B.; CHEN, J. D. Activation and Binding of Peroxisome ProliferatorActivated Receptor ã by Synthetic Cannabinoid Ajulemic Acid. Molecular Pharmacology, v. 63 n. 5, p. 983-992, 2003.

LOTTENBERG, A.M.P. Importância da gordura alimentar na prevenção e no controle de distúrbios metabólicos e da doença cardiovascular. Arquivos Brasileiros de Endocrinologia \& Metabologia, v. 53, n. 5, p. 595607, 2009.

LOTTENBERG, S.A.; GLEZER, A.; TURATTI, L.A. Metabolic syndrome: identifying the risk factors. Jornal de Pediatria, v. 83, n. 5, p. 204-208, 2007.

MA, L.; GAO, H.G.; LI, B.Y.; MA, Y.B.; YOU, B.A.; ZHANG, F.L. Grape seed Proanthocyanidin extracts inhibit vascular cell adhesion molecule expression induced of by advanced glycation endproducts through activation of peroxisome proliferators-activated receptor ã. Journal of Cardiovascular Pharmacology, v.49, n. 5, p. 293298, 2007.

MACHADO, U.F.; SCHAAN, B.D.; SERAFHIM, P.M. Transportadores de Glicose na Síndrome Metabólica. Arquivos Brasileiros de Endocrinologia \& Metabologia, v. 50, n. 2, p. 177-189, 2006.

MAYNADIER, M.; BASILE, I.; GARY-BOBO, M. Adiponectin normalization: a clue to the anti-metabolic syndrome action of rimonabant. Drug Discovery Today, v. 14, n. 3-4, p. 192-197, 2009.

MICHALIK, L.;AUWERX, J.; BERGER, J.P.; CHATTERJEE, V.K.; GLASS, C.K.; GONZALEZ F.J.; GRIMALDI, P.A.; KADOWAKI, T.; LAZAR, M.A.; O'RAHILLY, S.; PALMER, C.N.; PLUTZKY, J.; REDDY, J.K.; SPIEGELMAN, B.M.; STAELS, B.; WAHLI, W. International Union of pharmacology. LXI. Peroxisome Proliferator-Activated Receptors. Pharmacological Reviews. V. 58, n. 4, p. 726-741, 2006.

MORIUCHI, A.; YAMASAKI, H.; SHIMAMURA, M.; KITA, A.; KUWAHARA, H.; FUJISHIMA, K.; SATOH, T.; FUKUSHIMA, K.; HAYAKAWA, T.; MIZUQUCHI, H.; NAGAYAMA, Y.; ABIRU, N.; KAWASAKI, E.; EQUCHI, $K$. Induction of human adiponectin gene transcription by telmisartan, angiotensin receptor blocker, independently on PPARã activation. Biochemical and Biophysical Research Communications, v.356, p. 1024-1030, 2007.

NAKATA, M.; YADA, T.; SOEJIMA, N.; MARUYAMA, I. Leptin promotes aggregation of human platelets via

Rev. Bras. PI. Med., Campinas, v.15, n.3, p.449-466, 2013. 
the long form of its receptor. Diabetes, v. 48, n. 2, p. 426-429, 1999.

NISSEN, S.E.; WOLSKI, K. Effect of rosiglitazone on the risk of myocardial infarction and death from cardiovascular causes. The New England. Journal of Medicine, v. 356, n. 24, p. 2457-2471, 2007.

O'SULLIVAN, S.E.; KENDALL, D.A. Cannabinoid activation of peroxisme proliferator-activated receptors: Potential for modulation of inflammatory disease. Immunobiology, v. 215, n. 8, p. 611-616, 2009.

O'SULLIVAN, S.E.; SUN, T.; BENNETT, A.J.; RANDALL, M.D.; KENDALL, D.A. Time-dependent vascular actions of cannabidiol in the rat aorta. European Journal of Pharmacology, v. 612, n. 1-3, p. 61-68, 2009.

PACKER, L.; SIES, H. Oxidative stress and inflammatory mechanisms in obesity, diabetes, and the metabolic syndrome. $1^{\circ}$ Edição. Londres: CRC Press, 2008. 332p.

PAPA, P.C.; VARGAS, A.M.; SILVA, J.L.; NUNES, M.T.; MACHADO, U.F. GLUT-4 protein is differently modulated during development of obesity in monosodium glutamate-treated mice. Life Science, v. 71, n. 16, p. 1917-1928, 2002.

PEREIRA, V.C. Atividade agonista do extrato de Tabebuia heptaphylla sobre os receptores proliferadores peroxissomais alfa (PPARã), beta/ delta (PPARâ/ä) e gama (PPARã). 2008. 109p. Dissertação (Mestrado), Pós-Graduação em Ciência da Saúde. Universidade de Brasília, Brasília, 2008.

PISCHON, T.; GIRMAN, C.J.; HOTAMISLIGIL, G.S.; RIFAI, N.; HU, F.B.; RIMM, E.B. Plasma adiponectin levels and risk of myocardial infarction in men. JAMA, v. 291, n. 14, p. 1730-1737, 2004.

RABE, K.; LEHRKE, M.; PARHOFER, K.G.; BROEDL, U.C. Adipokines and insulin resistance. Molecular Medicine, v. 14, n. 11-12, p. 741-751, 2008.

REAVEN, G.M. Role of insulin resistance in human disease. Diabetes, v. 37, n. 12, p. 1595-1607, 1988.

SÁNCHEZ-MUÑOZ, F.; GARCÍA-MACEDO, R.;
ALARCÓN-AGUILAR, F.; CRUZA, M. Adipocinas, tejido adiposo y su relación com células Del sistema inmune. Gaceta Médica de México, v. 141, n. 6, p. 505-512, 2005.

SATO, M.; TAI, T.; NUNOURA, Y.; YAJIMA, Y.; KAWASHIMA, S.; TANAKA, K. Dehydrotrametenolic acid induces preadipocyte differentiation and sensitizes animal models of noninsulin-dependent diabetes mellitus to insulin. Biology and Pharmaceutical Bulletin, v. 25, n. 1, p. 81-86, 2002.

SHEARER, B.G.; BILLIN, A.N. The next generation of PPAR drugs: Do we have the tools to find them? Biochimica et Biophysica Acta, v. 1771, n. 8, p. 1082-1093, 2007.

SHEN, P.; LIU, M.H.; NG, T.Y.; CHAN, Y.H.; YONG, E.L. Differential effects os isoflavones, from Astragalus Membranaceus and Pueraria Thomsonii, on the activation of PPARá, PPARã and adipocyte differentiation in vitro. The Journal of Nutrition, v. 136, n. 4, p. 899-905, 2006.

TAKAHASHI, N.; KAWADA, T.; GOTO, T.; YAMAMOTO, T.; TAIMATSU, A.; MATSUI, N.; KIMURA, K.; SAITO, M.; HOSOKAWA, M.; MIYASHITA, K.; FUSHIKI, T. Dual action of isoprenols from herbal medicines on both PPARgamma and PPARalpha in 3T3-L1 adipocytes and HepG2 hepatocytes. FEBS Letters, v. 514, n. 2-3, p. 315-322, 2002.

UKKOLA, O.; SANTANIEMIM, M. Adiponectin: a link between excess adiposity and associated comorbidites? Journal Molecular Medicine, v. 80, n. 11, p. 696-702, 2002.

WILKINSON, A. S.; MONTEITH, G.R.; SHAW, P. N.; LIN, C-N.; GIDLEY, M. J., ROBERTS-THOMSON, S. J. Effects of the Mango Components Mangiferin and Quercetin and the Putative Mangiferin Metabolite Norathyriol on the Transactivation of Peroxisome Proliferator-Activated Receptor Isoforms. Journal of Agricultural and. Food Chemistry, v. 56, n. 9, p.3037-3042, 2008.

Rev. Bras. PI. Med., Campinas, v.15, n.3, p.449-466, 2013. 